\title{
The wall shear stress produced by the normal impingement of a jet on a flat surface
}

\author{
By DENIS J. PHARES ${ }^{1} \dagger$, GREGORY T. SMEDLEY ${ }^{1}$ \\ AND RICHARD C. FLAGA $\mathrm{N}^{2}$ \\ ${ }^{1}$ Environmental Engineering Science Department, California Institute of Technology, Pasadena, \\ CA 91125, USA \\ ${ }^{2}$ Chemical Engineering Department, California Institute of Technology, Pasadena, CA 91125, USA
}

(Received 24 May 1999 and in revised form 10 April 2000)

A method for the theoretical determination of the wall shear stress under impinging jets of various configurations is presented. Axisymmetric and two-dimensional incompressible jets of a wide range of Reynolds numbers and jet heights are considered. Theoretical predictions from this approach are compared with available wall shear stress measurements. These data are critically evaluated based on the method of measurement and its applicability to the boundary layer under consideration. It was found that impingement-region wall shear stress measurements using the electrochemical method in submerged impinging liquid jets provide the greatest accuracy of any indirect method. A unique wall shear stress measurement technique, based on observing the removal of monosized spheres from well-characterized surfaces, was used to confirm the impinging jet analysis presented for gas jets. The technique was also used to determine an empirical relation describing the rise in wall shear stress due to compressibility effects in impinging high-velocity jets.

\section{Introduction}

Impinging gas jets have most commonly been exploited for their enhanced heat and mass transfer characteristics when applied to industrial processes. Consequently, impinging jet research has generally focused on understanding and quantifying heat and mass transfer to a surface for a wide variety of impinging jet configurations. The present work is concerned with an often overlooked aspect of the flow - the wall shear stress produced on the surface. Recently, a few applications that utilize the shear imposed at the surface by impinging jets have been discussed in the literature. Deshpande \& Vaishnav (1982) used submerged impinging saline jets to probe the shear strength of the endothelial surface of a vascular tissue. The structure of this delicate layer of cells can be visibly altered by excessive applied shear. Tu \& Wood (1996) and Bouainouche, Bourabaa \& Desmet (1997) described a process known as jet stripping in which impinging plane jets are used to control liquid coating thickness on continuous metal strips. Impinging jets can also be used to extract particles from surfaces either as a cleaning method or to collect samples for chemical analysis, e.g. for detection of explosives or other contraband (Phares et al. 2000a). Smedley, Phares \& Flagan (1999) explored the entrainment of uniformly sized spherical particles by a normally

$\dagger$ Present address: Mechanical Engineering Department, University of Delaware, Newark, DE 19716, USA. 
impinging gas jet. The transition from complete adhesion to complete entrainment was abrupt, indicating that micron-sized particles $\left(D_{p}=8.3 \mu \mathrm{m}\right)$ could be used effectively as quantized shear stress sensors once calibrated with an appropriate experiment. This paper presents a theoretical and experimental study aimed at obtaining a reliable wall shear stress map for a wide range of jet Reynolds numbers and jet-to-plate spacings. In light of the aforementioned applications, both two-dimensional and axisymmetric jets will be considered, and we will restrict ourselves to the normal impingement of unconfined jets onto a flat surface.

Previous theoretical descriptions of the impinging jet flow field have involved two main schemes. The first entails numerically solving the full Navier-Stokes equations for the entire flow region. Several such studies have included calculations for the wall shear stress distribution. Deshpande \& Vaishnav (1982) considered the impinging laminar axisymmetric jet. Looney \& Walsh (1984) and Bouainouche et al. (1997) considered the impinging turbulent plane jet. The second approach to describing impinging jet flow fields involves separating the flow into regions where distinct approximations of the Navier-Stokes equations are valid (Strand 1964; Scholtz \& Trass 1970; Rubel 1980, 1983). This approach is more desirable for our purposes, since we are mainly concerned with the small region of flow located just above the impingement plane, where boundary layer approximations can be made. The analysis presented in $\S 2$ divides the flow into four regions: the free-jet region, the inviscid impingement region, the impingement boundary layer, and the wall-jet region. Flow solutions in the free jet and inviscid impingement regions provide the free-stream conditions for solution of the boundary layer equations needed to determine the wall shear stress in the impingement region. Farther from the stagnation point, as strong pressure gradients vanish, wall shear stress trends should approach those predicted by previous wall jet analyses (Glauert 1956; Poreh, Tsuei \& Cermak 1967; Lauder \& Rodi 1983).

Only a few of the many experimental investigations of impinging jets have included measurements of the wall shear stress, but the disagreement between investigators is higher than the reported uncertainties. In $\S 3$, we use the model developed in $\S 2$ to explore the cause of this disagreement and examine the influence of measurement method. In $\S 4$, we present wall shear stress measurements under an impinging circular jet using the measurement technique described by Smedley et al. (1999), which involves monitoring the removal of monodisperse microspheres from the impingement surface. Results from these experiments confirm the incompressible model from $\S 2$, and allow the development of an empirical correction factor based on the jet Mach number, $M$, for impinging compressible jets.

\section{Theoretical analysis}

Dividing impinging jet flow into separate regions was suggested by Bradshaw \& Love (1961), who stated that, since flow redirection occurred in such a small region, an inviscid impingement solution coupled with a boundary layer solution might accurately describe the flow. Scholtz \& Trass (1970) applied this method to the shortrange impingement of a laminar axisymmetric jet and made accurate predictions for mass transfer in the impingement region. Kataoka \& Mizushina (1974) used empirical free-stream conditions and a laminar boundary layer analysis at the surface to accurately predict wall shear stress in the impingement region of an axisymmetric impinging turbulent jet. The objective in this section is to generalize these approaches to include the effect of the free-jet velocity profile on the inviscid solution and the transition to turbulence in the boundary layer. 


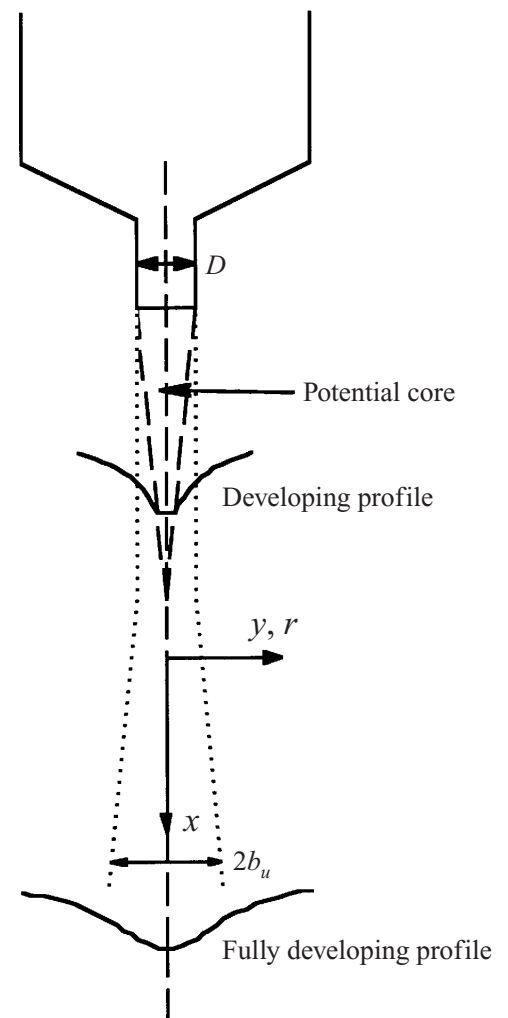

Figure 1. Schematic of the free jet region.

\subsection{The free-jet region}

A free jet consists of a near-field region, where a potential core has not yet experienced turbulent mixing with the quiescent fluid, and a far-field region, where the jet may be treated as a point source (or line source in the case of the plane jet) and the mean velocity profiles become similar (see figure 1). Mean velocity similarity generally occurs at a downstream distance of about 8 jet diameters. The wall pressure distribution produced by an impinging jet depends on the mean velocity profile of the fluid at the top of the inviscid impingement region - taken to be the surface of departure from the free-jet profile due to the effect of the wall. To maximize the range of applicability of the present work, both near-field and far-field mean velocity profiles for two-dimensional and axisymmetric free jets must be considered for the ensuing inviscid analysis.

\subsubsection{The far-field free-jet region}

Since practical applications of impinging jets do not involve very small jet Reynolds numbers, we employ Schlichting's (1960) far-field solution for the laminar free jet, wherein a constant longitudinal jet momentum is assumed. The far-field axial velocity profiles are

$$
\begin{gathered}
u(x, y)=0.4543\left(\frac{K^{2}}{v x}\right)^{1 / 3}\left(1-\tanh ^{2} \xi\right), \\
\xi=0.2752\left(\frac{K^{1 / 3}}{v^{2 / 3}}\right) \frac{y}{x^{2 / 3}}
\end{gathered}
$$




\begin{tabular}{|c|c|c|}
\hline & Velocity half-width & Centreline velocity \\
\hline Two-dimensional laminar & $b_{u} / x=3.20 R e_{o}^{-2 / 3}(x / D)^{-1 / 3}$ & $u_{m} / u_{o}=0.454 R e_{o}^{1 / 3}(x / D)^{-1 / 3}$ \\
\hline Axisymmetric laminar & $b_{u} / x=4.67 R e_{o}^{-1}$ & $u_{m} / u_{o}=0.0938 \operatorname{Re}_{o}(x / D)^{-1}$ \\
\hline Two-dimensional turbulent & $b_{u} / x=0.881 / \sigma$ & $u_{m} / u_{o}=0.866 \sigma^{1 / 2}(x / D)^{-1 / 2}$ \\
\hline Axisymmetric turbulent & $b_{u} / x=5.27 \epsilon_{0} / K^{1 / 2}$ & $u_{m} / u_{o}=0.106 K^{1 / 2} / \epsilon_{0}(x / D)^{-1}$ \\
\hline
\end{tabular}

for the two-dimensional jet, and

$$
\begin{gathered}
u(x, r)=\frac{3}{8 \pi} \frac{K}{v x}\left(1+\frac{1}{4} \eta^{2}\right)^{-2}, \\
\eta=\frac{1}{4}\left(\frac{3}{\pi}\right)^{1 / 2} \frac{K^{1 / 2}}{v} \frac{r}{x}
\end{gathered}
$$

for the axisymmetric jet, where $v$ and $K$ are the kinematic viscosity and jet momentum, respectively. As shown in figure 1, the coordinate system is chosen such that $x$ is oriented downstream along the jet axis, and $y$ and $r$ are oriented perpendicularly outward from the jet centreline for the two-dimensional and axisymmetric jet, respectively. The laminar solution is accurate only for jet Reynolds numbers under 30 (Looney \& Walsh 1984). Extending the axisymmetric development to the turbulent jet simply involves replacing the kinematic viscosity, $v$, with the virtual kinematic viscosity, $\epsilon_{0}$ (Schlichting 1960).

The far-field mean axial velocity profile for the two-dimensional turbulent jet is

$$
\begin{gathered}
u(x, y)=\frac{\sqrt{3}}{2}\left(\frac{K \sigma}{x}\right)^{1 / 2}\left(1-\tanh ^{2} \zeta\right), \\
\zeta=\frac{\sigma y}{x},
\end{gathered}
$$

where $\sigma$ is an empirical constant.

In addition to providing the influx conditions to the inviscid impingement region for larger jet-to-surface separations, these similar velocity profiles also possess convenient length and velocity scaling factors in the velocity half-width, $b_{u}$, and the centreline velocity, $u_{m}$. Table 1 lists these quantities in terms of the jet exit conditions, where $D$ is the nozzle size (diameter for a circular jet, and slit width for a plane jet), $u_{o}$ is the jet exit velocity, and $R e_{o}=u_{o} D / v$ is the jet Reynolds number. Accepted values for the empirical constants, $\sigma$ and $\epsilon_{0} / K^{1 / 2}$, are 7.5-7.7 (Schlichting 1960; Looney \& Walsh 1984) and 0.016-0.018 (Schlichting 1960; Bradbury 1972; Beltaos \& Rajaratnam 1974), respectively.

\subsubsection{The near-field free-jet region}

Close to the jet nozzle, the development of the turbulent mixing zone between the potential core and the quiescent fluid must be considered. For a uniform velocity at the jet exit, the mixing zone mean velocity profile in a two-dimensional jet is described 


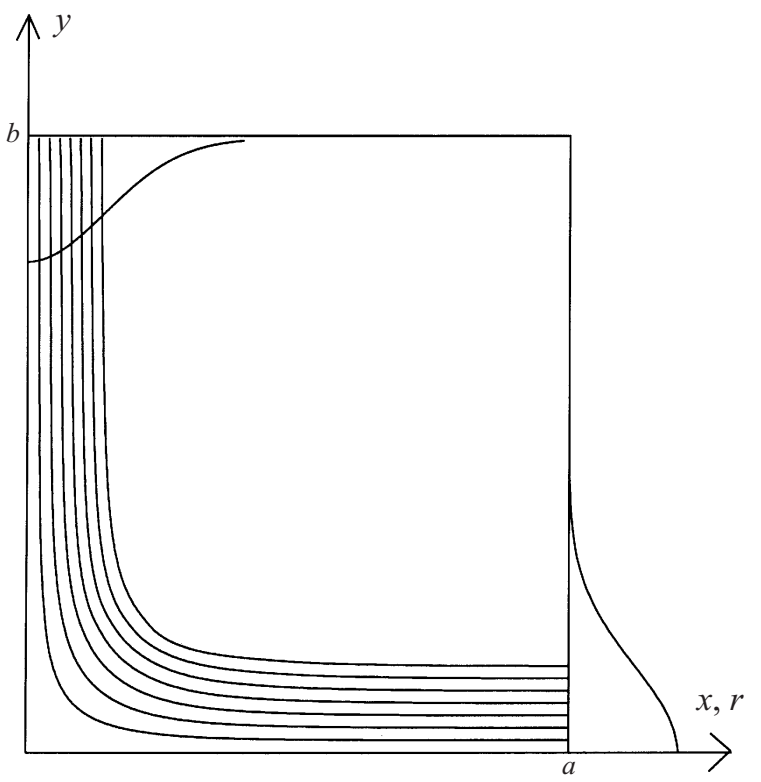

FIGURE 2. Schematic of the inviscid impingement region.

well by Tollmein's solution (Abramovich 1963):

$$
\begin{gathered}
\frac{u}{u_{o}}=0.0176 \exp \left(-\phi_{1}\right)+0.1337 \exp \left(\frac{\phi_{1}}{2}\right) \cos \left(\frac{\sqrt{3}}{2} \phi_{1}\right) \\
+0.6876 \exp \left(\frac{\phi_{1}}{2}\right) \sin \left(\frac{\sqrt{3}}{2} \phi_{1}\right), \\
\phi_{1}=\frac{1}{\sigma^{\prime}}\left(\frac{0.5 D-y}{x}\right),
\end{gathered}
$$

where the empirical constant is given by $\sigma^{\prime}=0.09$. In this case, the jet half-width, $b_{u}$, grows very slightly with downstream distance:

$$
\frac{b_{u}}{D}=0.5+0.03 \frac{x}{D} \text {. }
$$

For the near-field axisymmetric jet, the mixing-zone mean velocity profile is described well by the empirical expression of Wall, Subramanian \& Howley (1982):

and

$$
\begin{gathered}
\frac{u}{u_{o}}=0.5\left(1+\cos \left(\pi \phi_{2}\right)\right), \\
\phi_{2}=\frac{r+0.12 x-0.5 D}{0.28 x}, \quad 0 \leqslant \phi_{2}<\frac{1}{2},
\end{gathered}
$$

$$
\frac{b_{u}}{D}=0.5+0.02 \frac{x}{D}
$$

\subsection{The inviscid impingement region}

The velocity profile of the oncoming fluid deviates from the free-jet result as the fluid approaches the surface. Within the small region bounded by this location and the 
top of the boundary layer on the surface, the streamlines are redirected from roughly perpendicular to parallel to the surface. Using the coordinate system shown in figure 2, the inviscid flow is described by the stream function-vorticity equation:

$$
\frac{\partial^{2} \psi}{\partial y^{2}}+\frac{\partial^{2} \psi}{\partial x^{2}}=\Omega(x, y)
$$

and

$$
\frac{\partial^{2} \psi}{\partial y^{2}}+\frac{\partial^{2} \psi}{\partial r^{2}}-\frac{1}{r} \frac{\partial \psi}{\partial r}=r^{2} \Omega(r, y),
$$

for two-dimensional and axisymmetric impingement, respectively. In (2.13), the stream and vorticity functions are defined:

and in (2.14):

$$
\begin{gathered}
\frac{\partial \psi}{\partial x}=-v, \quad \frac{\partial \psi}{\partial y}=u, \\
\Omega=\frac{\partial u}{\partial y}-\frac{\partial v}{\partial x},
\end{gathered}
$$

$$
\begin{gathered}
\frac{\partial \psi}{\partial r}=-r v, \quad \frac{\partial \psi}{\partial y}=r u, \\
\Omega=\frac{1}{r}\left(\frac{\partial u}{\partial y}-\frac{\partial v}{\partial r}\right) .
\end{gathered}
$$

Defining the stream function such that $\psi=0$ along the surface and centreline and assuming parallel outflow far from the stagnation point provides three boundary conditions. The fourth is determined by the influx velocity profile. It is apparent from figure 1 that a variety of impinging velocity profiles are possible depending on the jet Reynolds number, $R e_{o}$, and the jet height, $H$, from the surface. Therefore, treatment of the inviscid impingement region must not be limited to a single velocity profile.

Phares, Smedley \& Flagan (2000b) solved (2.13) and (2.14) for an arbitrary influx stream function profile, $\Psi$, in terms of a surface integral involving the vorticity function, $\Omega$. By assuming an appropriate stream function distribution, the vorticity function and, thus, the surface integral could be calculated, yielding a corrected stream function distribution. For the purpose of calculating wall shear stress, the significant quantity needed from the inviscid analysis is the velocity, $U$, at the surface $y=0$, which is assumed to be the velocity at the top of the boundary layer. It follows from the development of Phares et al. (2000b) that this velocity distribution is the converging infinite series:

$$
\begin{gathered}
\frac{U(x)}{u_{s}}=\sum_{n=1}^{\infty}\left[A_{n} \gamma_{n}-\sum_{m=1}^{\infty} \frac{B_{m n} \alpha_{m}}{\gamma_{n}^{2}+\alpha_{m}^{2}}\right] \sin \left(\gamma_{n} x\right), \\
A_{n}=\frac{2}{a \sinh \left(\gamma_{n} b\right)} \int_{0}^{a} \Psi\left(x^{\prime}\right) \sin \left(\gamma_{n} x^{\prime}\right) \mathrm{d} x^{\prime}, \\
B_{m n}=\frac{4}{a b} \int_{0}^{a} \int_{0}^{b} \Omega\left(x^{\prime}, y^{\prime}\right) \sin \left(\alpha_{m} y^{\prime}\right) \sin \left(\gamma_{n} x^{\prime}\right) \mathrm{d} y^{\prime} \mathrm{d} x^{\prime}, \\
\gamma_{n}=\frac{2 n-1}{2 a} \pi, \quad \alpha_{m}=\frac{m \pi}{b},
\end{gathered}
$$


for the two-dimensional case, and

$$
\begin{gathered}
\frac{U(r)}{u_{s}}=\sum_{n=0}^{\infty}\left[D_{n} \lambda_{n}-\sum_{m=0}^{\infty} \frac{E_{m n} \alpha_{m}}{\lambda_{n}^{2}+\alpha_{m}^{2}}\right] r J_{1}\left(\lambda_{n} r\right), \\
D_{n}=\frac{2}{a^{2} J_{1}^{2}\left(\lambda_{n} a\right) \sinh \left(\lambda_{n} b\right)} \int_{0}^{a} \Psi\left(r^{\prime}\right) J_{1}\left(\lambda_{n} r^{\prime}\right) \mathrm{d} r^{\prime}, \\
E_{m n}=\frac{4}{b a^{2} J_{1}^{2}\left(\lambda_{n} a\right)} \int_{0}^{a} \int_{0}^{b} r^{\prime 2} \Omega\left(r^{\prime}, y^{\prime}\right) J_{1}\left(\lambda_{n} r^{\prime}\right) \sin \left(\alpha_{m} y^{\prime}\right) \mathrm{d} y^{\prime} \mathrm{d} r^{\prime}, \\
J_{0}\left(\lambda_{n} a\right)=0,
\end{gathered}
$$

for the axisymmetric case. In equations (2.19) and (2.23), $u_{s}$ is the centreline velocity of the influx flow and $a$ and $b$ are the boundary locations chosen far enough away from the stagnation point so as to not affect the solution. Equations (2.19) and (2.23) are compared with available measurements for near-field and far-field jet impingement in figures 3 and 4, respectively. The experimental data presented were calculated from Bernoulli's equation using surface pressure measurements. For fully developed jet impingement (figures $3 a$ and $4 a$ ) the data collapse to the inviscid solution when scaled with $b_{u}$. For developing jet impingement, the inviscid theory predicts a noticeable effect of jet height on the surface velocity for the two-dimensional case (figure $3 b$ ) and the axisymmetric case (figure $4 b$ ). The experimental data support these predictions.

\subsection{The impingement boundary layer}

Having obtained the free-stream conditions for a wide range of impinging jet configurations, we can now proceed with the boundary layer analysis that will enable the determination of the wall shear stress distribution on the surface. Theoretical treatment of the boundary layer requires some initial intuition about the flow. It could be inferred that the strong favourable pressure gradients close to the stagnation point not only surpress turbulence close to the surface, but also cause the boundary layer to at least behave in a laminar fashion. This inference is strongly supported by the work of Kataoka \& Mizushina (1974) and Kataoka et al. (1982), who observed that, in contrast to heat and mass transfer, the wall shear stress close to the stagnation point is insensitive to the level of turbulence in the free stream. As the pressure gradients subside, we expect the boundary layer to become turbulent. The location of this transition will be explored using the available data later.

\subsubsection{Laminar boundary layer}

Following Polhausen (Schlichting 1960), the wall shear stress, $\tau_{o}$, in a laminar boundary layer subject to a pressure gradient is given by

$$
\frac{\tau_{o} \delta}{\mu U}=2+\frac{\Lambda}{6}
$$

where $\delta$ is the boundary layer thickness, and $\Lambda$ is a boundary layer velocity profile shape factor defined as

$$
\Lambda=\frac{\delta^{2}}{v} \frac{\mathrm{d} U}{\mathrm{~d} x}
$$



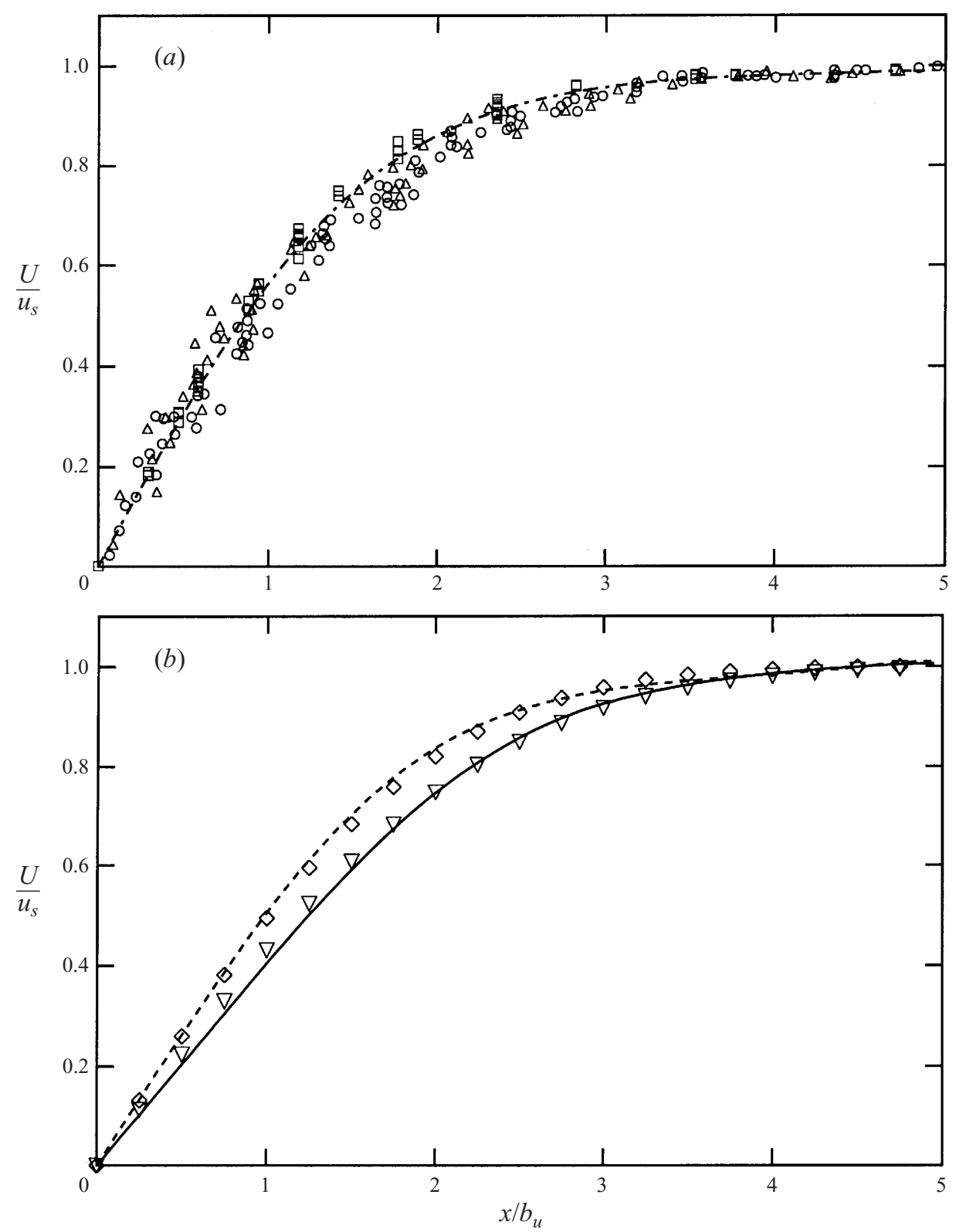

FIGURE 3. Comparison between calculated (equation (2.19)) surface velocities with influx boundary condition given by equations (2.5) $(---)$ and $(2.7), H / D=1(-), H / D=5(---)$, and impinging plane jet measurements. (a) Fully developed turbulent jet impingement $(H / D>6)$ : Schauer \& Eustis $(\square)$; Kumada \& Mabuchi $(\triangle)$; Beltaos \& Rajaratnam (O). (b) Developing jet impingement $(H / D<6)$ : Tu \& Wood, $H / D=1(\nabla)$ and $H / D=4(\diamond)$.

Calculation of $\delta$ and $\Lambda$ by the method of Holstein and Bohlen is detailed by Schlichting (1960) for the two-dimensional case and by Kataoka \& Mizushina (1974) for the axisymmetric case and is thus not presented here.

It follows from equations (2.27) and (2.28) that the wall shear stress distribution 
Normal shear stress produced by impingement of a jet
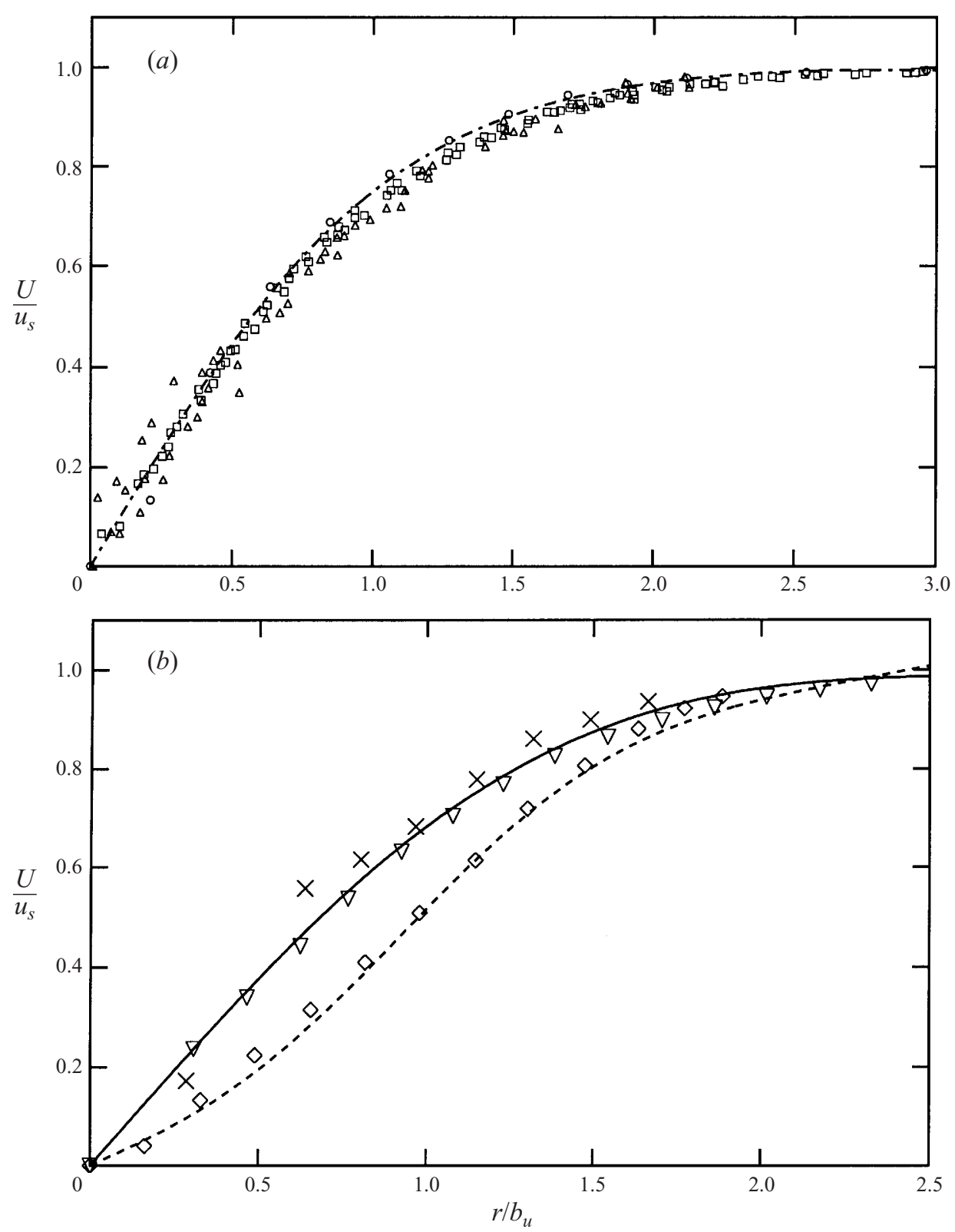

Figure 4. Comparison between calculated (equation (2.23)) surface velocities with influx boundary condition given by equations (2.3) $(---)$ and (2.10), $H / D=6(-), H / D=1.2(--)^{-}$, and impinging circular jet measurements. (a) Fully developed turbulent jet impingement $(H / D \geqslant 8)$ : Bradbury (O); Beltaos \& Rajaratnam $(\triangle)$; Giralt, Chia \& Trass ( $\square$ ). (b) Developing jet impingement $(H / D<8)$ : Giralt et al., $H / D=6(\nabla)$ and $H / D=1.2(\diamond)$; Kataoka et al., $H / D=6(\times)$.

can be written in the universal form

$$
\frac{\tau}{\rho u_{s}^{2}}\left(\frac{u_{s} b_{u}}{v}\right)^{1 / 2}=g\left(\frac{x}{b_{u}}\right),
$$

for fully developed turbulent jet impingement, where $x$ is replaced by $r$ for the 

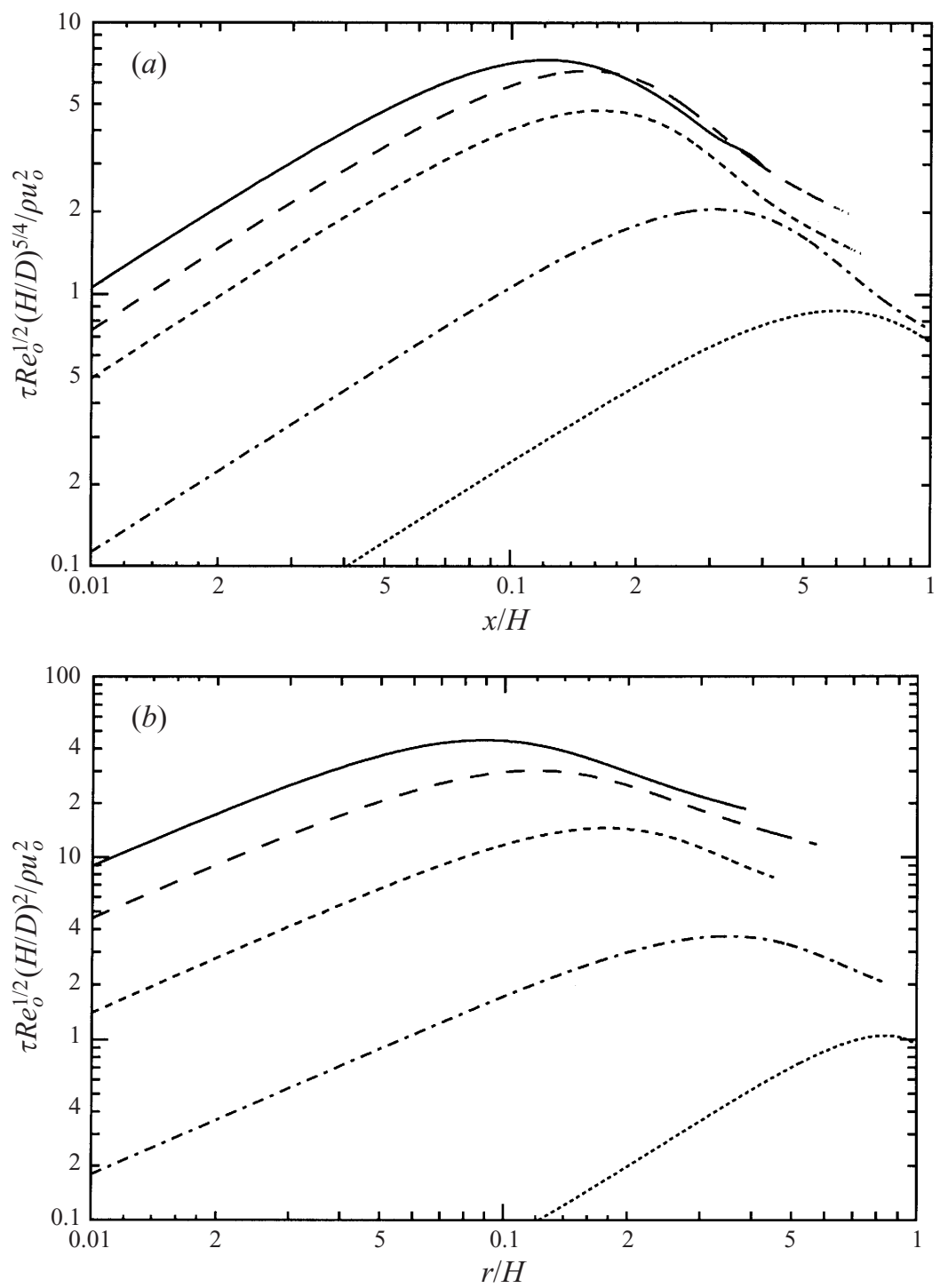

FIGURE 5. Predicted wall shear stress distributions based on laminar boundary layer theory for $H / D \geqslant 8(-), H / D=6(--), H / D=4(---), H / D=2(-\cdot-\cdot)$, and $H / D=1(\cdots \cdots)$ for: (a) plane jet impingement; $(b)$ circular jet impingement.

axisymmetric case. Using this scaling and table 1, the wall shear stress can be scaled in terms of the jet nozzle parameters yielding the universal functions $g_{1}$ and $g_{2}$ :

$$
\frac{\tau}{\rho u_{o}^{2}} \operatorname{Re}_{o}^{1 / 2}\left(\frac{H}{D}\right)^{5 / 4}=g_{1}\left(\frac{x}{H}\right)
$$

for the two-dimensional case, and

$$
\frac{\tau}{\rho u_{o}^{2}} R e_{o}^{1 / 2}\left(\frac{H}{D}\right)^{2}=g_{2}\left(\frac{r}{H}\right)
$$

for the axisymmetric case. These functions are plotted in figure 5 for two-dimensional 
and axisymmetric fully developed jet impingement along with the non-similar wall shear stress distributions resulting from developing jet impingement $(H / D<8)$. The magnitude, $\tau_{m}$, and location, $x_{m}\left(r_{m}\right)$, of the shear stress maximum for fully developed jet impingement are

$$
\tau_{m}=7.30 \rho u_{o}^{2} R e_{o}^{-1 / 2}\left(\frac{H}{D}\right)^{-5 / 4}, \quad \frac{x_{m}}{H}=0.12
$$

and

$$
\tau_{m}=44.6 \rho u_{o}^{2} R e_{o}^{-1 / 2}\left(\frac{H}{D}\right)^{-2}, \quad \frac{r_{m}}{H}=0.09
$$

for two-dimensional and axisymmetric impingement, respectively.

\subsubsection{Turbulent boundary layer}

The transition from a laminar to a turbulent boundary layer within the impingement region can be accompanied by a sharp increase in the wall shear stress as observed by Kataoka \& Mizushina (1974) and Alekseenko \& Markovich (1994) (see §3). The location and magnitude of this increase is variable within their experiments. In the following boundary layer analysis, the magnitude of the increase will be calculated in terms of the transition location, $r_{t}$. Since the available measurements involve circular jets, only the axisymmetric case will be considered here.

Schlichting (1960) showed that the axisymmetric momentum equation could be written

$$
U^{2} \frac{\mathrm{d} \theta}{\mathrm{d} r}+(H+2) \frac{\theta}{U} \frac{\mathrm{d} U}{\mathrm{~d} r}+\frac{\theta}{r}=\frac{\tau}{\rho U^{2}},
$$

where $\theta$ is the momentum thickness, and the ratio, $H$, of the displacement and momentum thicknesses is generally taken to be equal to 1.4 . The shear stress, $\tau$, can be related to $\theta$ for turbulent boundary layers:

$$
\frac{\tau}{\rho U^{2}}=\alpha\left(\frac{U \theta}{v}\right)^{1 / n},
$$

where the constants were given by V. M. Falkner to be $\alpha=0.0065$ and $n=6$ (Schlichting 1960). Combining equations (2.34) and (2.35) and integrating directly yields

$$
\theta=\left[0.0076 v^{1 / 6} U^{-3.97} r^{-7 / 6}\left(C+\int_{r_{t}}^{r} r^{7 / 6} U^{3.80} \mathrm{~d} r\right)\right]^{6 / 7} .
$$

The constant of integration, $C$, is determined from the laminar result at $r_{t}$.

Figure 6 depicts the wall shear stress distribution for fully developed jet impingement assuming three different turbulence transition locations, $r_{m} / H=0.2,0.3$, and 0.4 . Based on this analysis, it appears that the magnitude of the shear stress jump will increase as the distance of the transition location from the stagnation point increases. This is consistent with the observation of Alekseenko \& Markovich (1994) that the turbulence transition is more pronounced for lower jet heights, which exhibit lower free-stream turbulence levels, and thus longer laminar boundary layer lengths from the stagnation point.

Soon after the transition to a turbulent boundary layer occurs, viscous dissipation results in a wall jet and the free-stream conditions obtained from inviscid theory are no longer accurate. Wall jets have been studied thoroughly both experimentally and theoretically (Launder \& Rodi 1983) and are, therefore, not considered in the present 


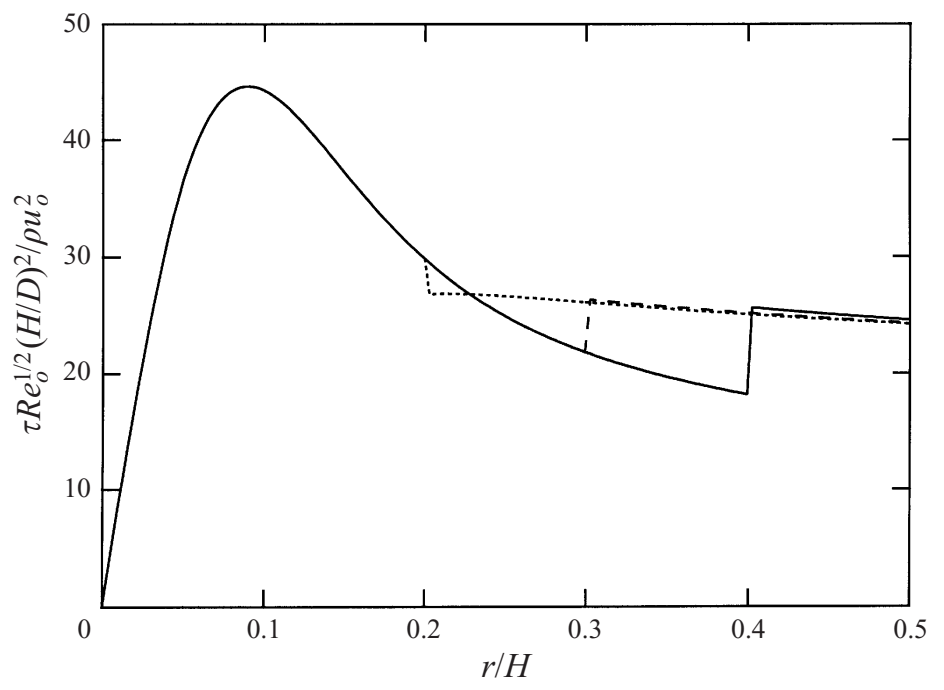

FIGURE 6. Wall shear stress distribution for three turbulence transition locations: $r_{t} / H=0.2(\cdots \cdots), r_{t} / H=0.3(--)$, and $r_{t} / H=0.4(-)$.

\begin{tabular}{|c|c|c|c|}
\hline Study & $H / D$ & $R e_{o}$ & Measurement technique \\
\hline Bradshaw \& Love (1961) & 18 & $1.50 \times 10^{5}$ & flat Preston tube \\
\hline \multicolumn{4}{|l|}{ Beltaos \& Rajaratnam (1974) } \\
\hline 'Run 4' & 21.1 & $8.04 \times 10^{4}$ & standard Preston tube \\
\hline 'Run 5' & 65.7 & $3.00 \times 10^{4}$ & \\
\hline Kataoka \& Mizushina (1974) & $3.86 \& 8.24$ & $9.60 \times 10^{3}-3.62 \times 10^{4}$ & electrochemical method \\
\hline Kataoka et al. (1982) & 6 & $4.00 \times 10^{3}-1.50 \times 10^{4}$ & electrochemical method \\
\hline Alekseenko \& Markovich (1994) & $2-8$ & $4.16 \times 10^{4}$ & electrochemical method \\
\hline
\end{tabular}

work. However, we will make use of the empirical expression of Poreh et al. (1967) for the mean shear stress in a radial wall jet,

$$
\frac{\tau}{\rho u_{o}^{2}} \operatorname{Re}_{o}^{1 / 2}\left(\frac{H}{D}\right)^{2}=0.34 R e_{o}^{1 / 5}\left(\frac{r}{H}\right)^{-2.3},
$$

in order to describe the circular jet data beyond the turbulence transition location.

\section{Wall shear stress measurements}

\subsection{Axisymmetric jets}

Table 2 lists available wall shear stress measurements under impinging axisymmetric jets. The most notable feature of these measurements is the agreement with laminar boundary layer theory of the wall shear stress distribution under the submerged impinging liquid jets (see figure 7), and the lack of agreement for the impinging gas jets (see figure 8). Figure 7 displays the wall shear stress measurements of Kataoka \& Mizushina (1974), Kataoka et al. (1982), and Alekseenko \& Markovich (1994) for various jet heights, $H$, and jet Reynolds numbers obtained using mass transfer techniques. In figures 7 and 8 , the radial location is non-dimensionalized with jet 

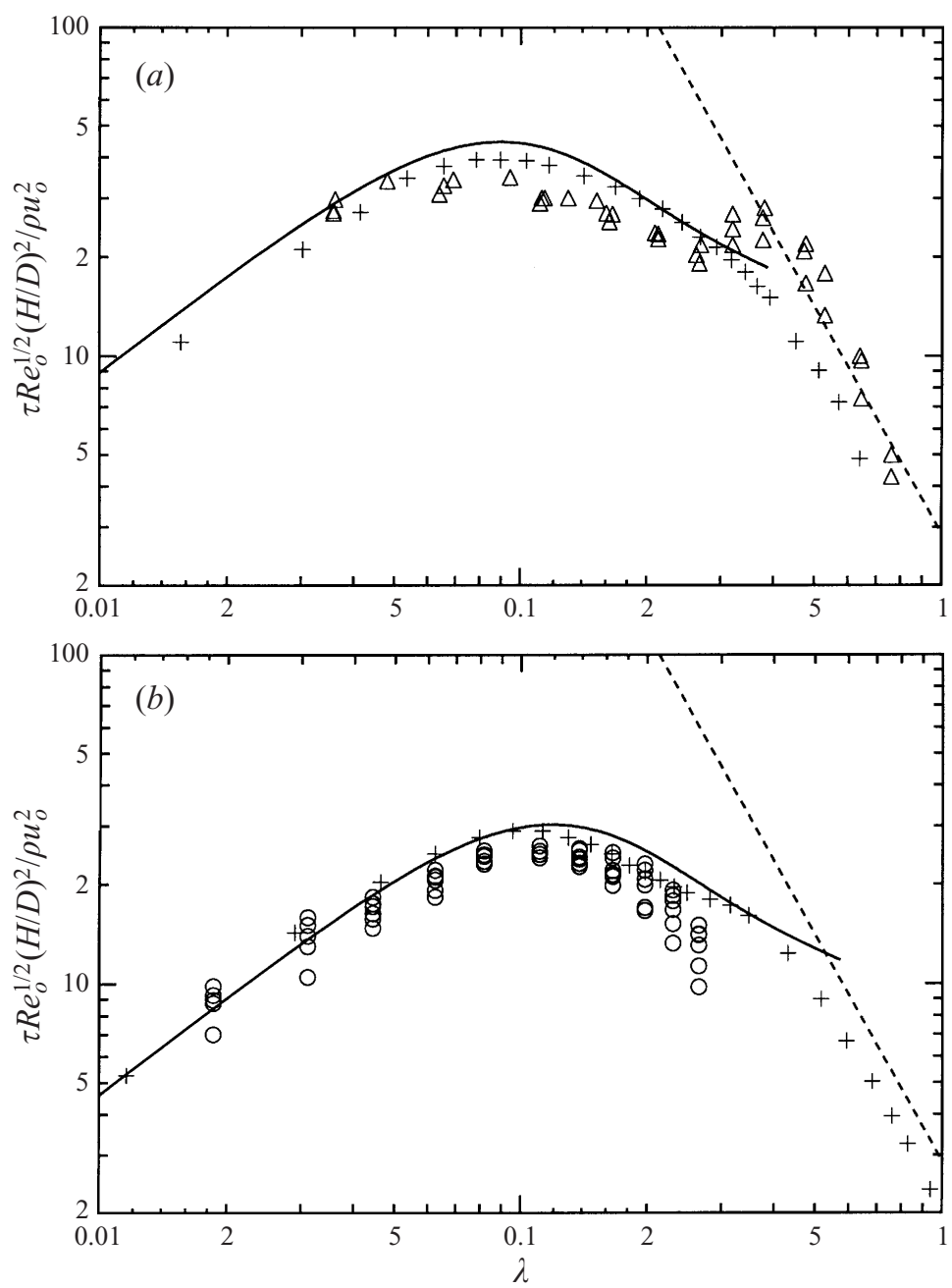

Figure 7. Wall shear stress measurements of Kataoka \& Mizushina (1974) $\left(R e_{o}=10600-36200\right)$ $(\triangle)$; Kataoka et al. (1982) $\left(R e_{o}=4000-15000\right)(0)$; and Alekseenko \& Markovich (1994) $\left(R e_{o}=41600\right)(+)$; compared with the impingement-region model $(-)$ and the wall-jet-region expression (equation (2.37)) (- - -) for $(a) H / D \geqslant 8 ;(b) H / D=6$.

height $(\lambda=r / H)$. Not only is the collapse to the predicted scaling for laminar flow clear, but the transition from laminar to turbulent boundary layer is evidenced by a sharp increase in shear stress in some of the data (see figure 7a).

In the case of the normally impinging axisymmetric gas jet, the only impingementregion wall shear stress data found in the literature were provided by Bradshaw \& Love (1961) for one jet configuration and Beltaos \& Rajaratnam (1974) for two jet configurations (labelled 'Run 4' and 'Run 5'). The latter compiled all three data sets, and non-dimensionalized the wall shear stress distributions with the maximum shear stress. The resulting curves appeared to collapse to a general curve determined from the axial boundary layer equation, and given by

$$
\frac{\tau}{\tau_{m}}=0.18\left(\frac{1-\mathrm{e}^{-114 \lambda^{2}}}{\lambda}\right)-0.9 .43 \lambda \mathrm{e}^{-114 \lambda^{2}},
$$




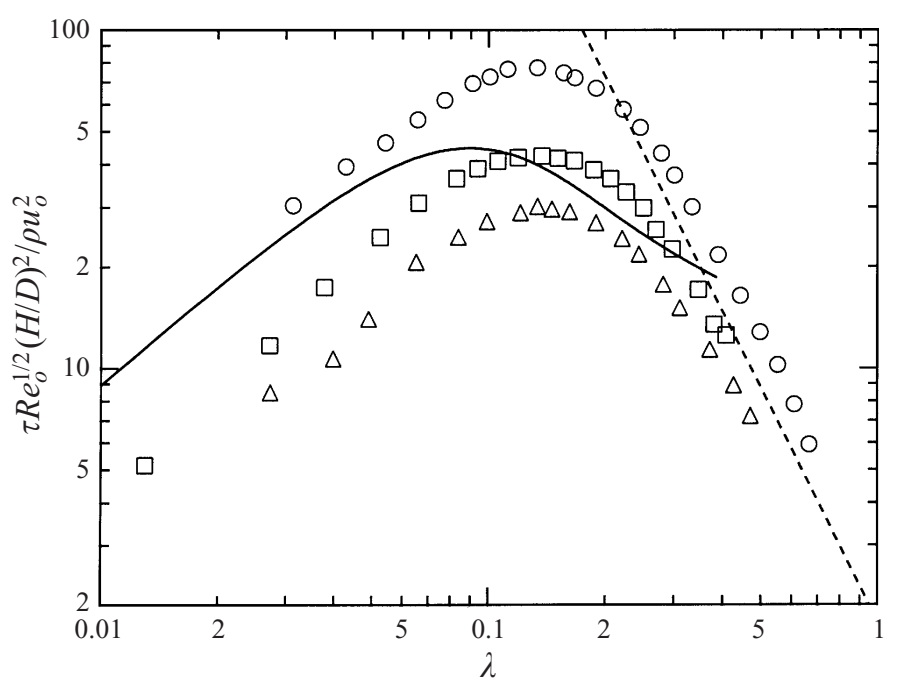

FIGURE 8. Wall shear stress measurements of Bradshaw \& Love $(1961)\left(R e_{o}=150000, H / D=18\right)$ (O); and Beltaos \& Rajaratnam (1974), Run $4\left(R e_{o}=80400, H / D=21.1\right)(\square)$; and Run $5\left(R e_{o}=30000, H / D=65.7\right)(\triangle)$; compared with impingement-region model $(\square)$ and wall-jet-region expression (equation (2.37)) (- - - -).

where $\lambda=r / H$ and the maximum shear stress, $\tau_{m}$, is given by

$$
\tau_{m}=0.16 \frac{\rho u_{o}^{2}}{(H / D)^{2}}
$$

The agreement between the data and this theoretical approach is misleading. Since the equation of motion was solved in the axial direction, the wall shear stress was erroneously defined as proportional to the radial variation in the radial velocity. Thus, the reported theoretical development and equation (3.1) are not valid. The correct approach would have been to solve the boundary layer equation in the radial direction along the surface, as in $\S 2.3$. Equation (3.2) is an empirical relation deduced from the three distributions, and appears to hold only to within $15 \%$ for these cases.

Figure 8 displays the three measured wall shear stress distributions along with the predictions from laminar boundary layer theory for the same jet parameters. It is curious that the measured shear stresses are not consistently higher or lower than the corresponding laminar predictions. In Run 4 from the experiments of Beltaos $\&$ Rajaratnam, laminar theory seems to overpredict the measured shear stress closer to the stagnation point and then switches to underpredicting at a non-dimensional radius of about 0.12. Laminar theory overpredicts the shear stresses from Run 5 and underpredicts the Bradshaw \& Love shear stresses throughout the impingement region.

An explanation of the differences observed between gas jet data and submerged liquid jet data may be derived from the manner in which the wall shear stress was measured. Kataoka \& Mizushina (1974), Kataoka et al. (1982), and Alekseenko \& Markovich (1994) used electrochemical probes and shear stress was calculated from the diffusion current. Bradshaw \& Love used a flat Preston tube of height $0.38 \mathrm{~mm}$, and Beltaos \& Rajaratnam used a standard Preston tube with an outside diameter of $1.2 \mathrm{~mm}$. In both cases, the probes were calibrated in fully developed turbulent shear flows. The use of Preston tubes to measure wall shear stress in pressure gradients was 

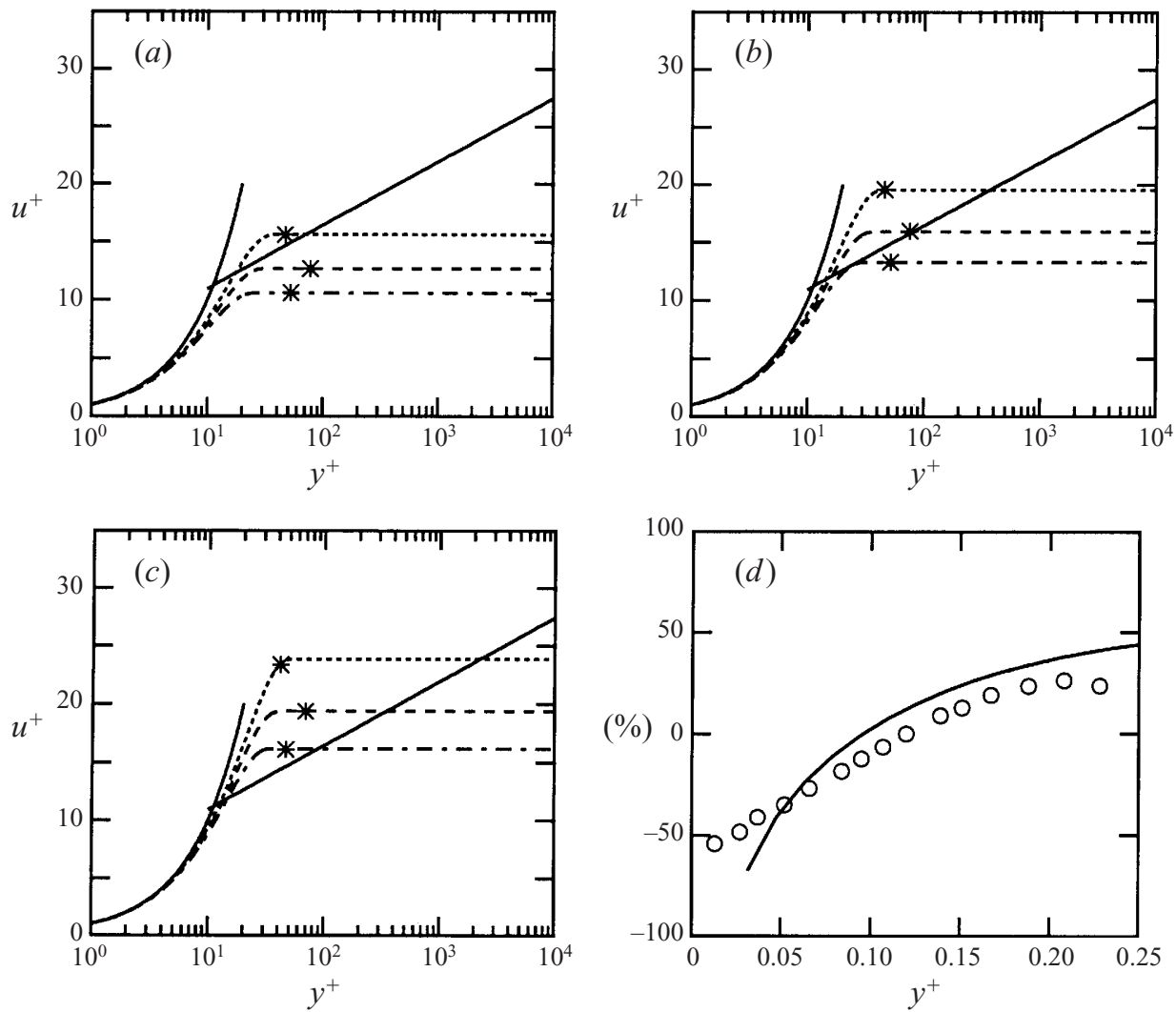

Figure 9. Turbulent boundary layer ( - ) compared to the location of the top of the Preston tube (*) in the laminar boundary layer under an impinging jet studied by Bradshaw \& Love (1961) $(\cdots \cdots)$ and Beltaos \& Rajaratnam (1974), Run $4\left(--_{-}\right)$and Run $5\left(-\cdot \cdot^{-}\right)$at radial locations of (a) $\lambda=0.08$, (b) $\lambda=0.12$, and (c) $\lambda=0.17$. (d) Correlation of relative shear stress measurement error for Run $4(\mathrm{O})$ and relative difference between assumed boundary layer profiles.

examined by Patel (1965), who found that laminarization of a turbulent boundary layer severely affects Preston tube accuracy in favourable pressure gradients. More recently, Champion \& Libby (1994) applied an asymptotic analysis to impinging jet flows and found stagnating turbulent flows to be significantly different close to the surface than turbulent shear flows. Since there is strong evidence from the submerged liquid jet experiments that the boundary layer in the impingement region is laminar, we would expect that the Preston tube cannot be used to measure shear stress within the impingement region of an impinging gas jet. Whether this holds for jets of larger Reynolds numbers or large jet heights - such as the three presented here - requires additional analysis of the boundary layer in conjunction with the available Preston tube data.

Patel found that reducing the diameter of the Preston tube counterintuitively increases the overestimate of the actual wall shear stress in a strong favourable pressure gradient. This can be attributed to the departure of the boundary layer profile from the logarithmic law for fully turbulent flows to a laminar profile. Figure $9(a-c)$ depicts the laminar velocity profiles, as calculated by the method of Holstein and Bohlen, for all three impinging jets at three non-dimensional radii. The symbols represent the location of the Preston tube within the boundary layer. Similar to the 


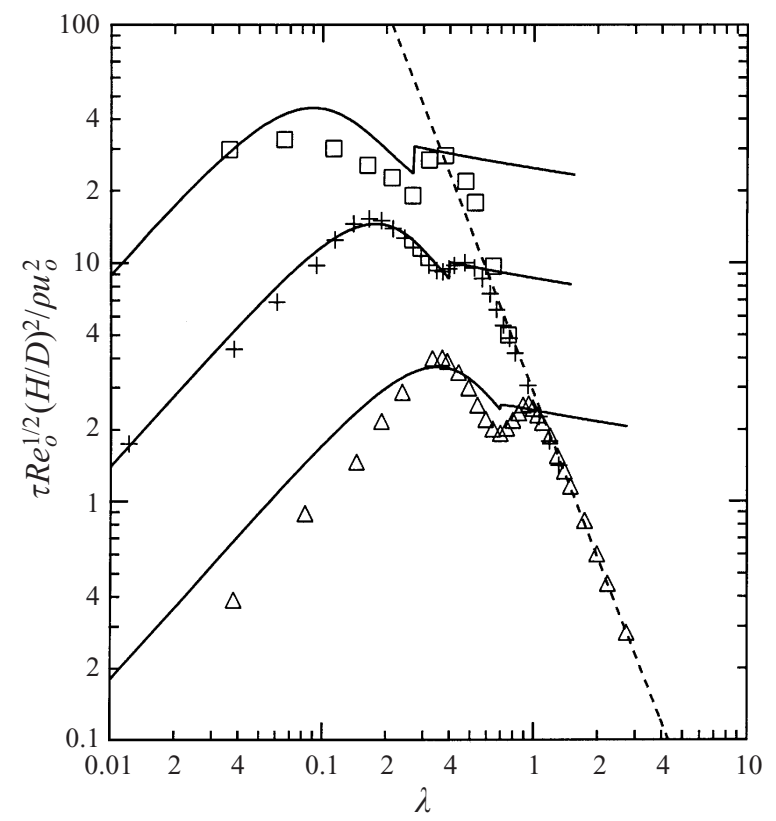

FIGURE 10. Transition to turbulence exhibited in three impinging jets: $\operatorname{Re}_{o}=21500, H / D=8$ ( $\square$ ) (Kataoka \& Mizushina 1974), $\operatorname{Re}_{o}=42600, H / D=4(+)$ and $H / D=2(\triangle)$ (Alekseenko \& Markovich 1994); compared with transition theory (equation (2.35)) (- ${ }_{-}$) and wall jet theory (equation (2.37)) (- - - ).

observations of Patel, it appears that the data of Beltaos \& Rajaratnam are more accurate because the deviations from the logarithmic law are not as great at the location prescribed by the size of the Preston tube, despite the fact that their tube is three times the size of the tube used by Bradshaw \& Love. Furthermore, there is correlation between the deviation of the measured shear stress from the laminar prediction and the difference between the laminar velocity profile and the logarithmic law at the Preston tube location. Figure $9(d)$ displays this loose correlation for Run 4. A better correlation could be achieved by considering the integral of the velocity over the entrance surface of the Preston tube rather than the velocity at the top of the tube; but the correlation presented is sufficient to demonstrate the high error that could result from using Preston tubes close to the stagnation point of an impinging jet.

The prominent shear stress rise indicative of the transition to a turbulent boundary layer appears under impinging jets with lower jet heights and jet Reynolds numbers. A few of these examples are displayed in figure 10 along with the predictions from $\S 2.3 .2$. The wall shear stress immediately after transition is described well by turbulent boundary layer theory, and quickly conforms to the radial wall jet expression (equation (2.37)).

\subsection{Plane jets}

Indirect measurements of wall shear stress under impinging plane jets (including the measurements of Schauer \& Eustis 1963, Beltaos \& Rajaratnam 1973, and Tu \& Wood 1996, see table 3) provide similar results to those presented for impinging circular jets and are not analysed here. However, one experiment run by Tu \& Wood (1996) may provide some additional insight into the form of the boundary layer close 
Study

Schauer \& Eustis (1963)

Beltaos \& Rajaratnam (1973)

Baines \& Keffer (1976)

Tu \& Wood (1996)

$$
H / D
$$

$40 \quad 4.3 \times 10^{4}$

$43.6 \& 66.1 \quad 5.3 \times 10^{3}-7.1 \times 10^{3}$

$3.86 \& 8.24 \quad 7.0 \times 10^{3}$

$1-21 \quad 3.04 \times 10^{3}-1.10 \times 10^{4}$

\section{Measurement technique}

flat Preston tube

flattened Preston tube

hot film, hot wire

Preston and Stanton tubes

TABLE 3. Impingement-region wall shear stress data for two-dimensional impinging jets.
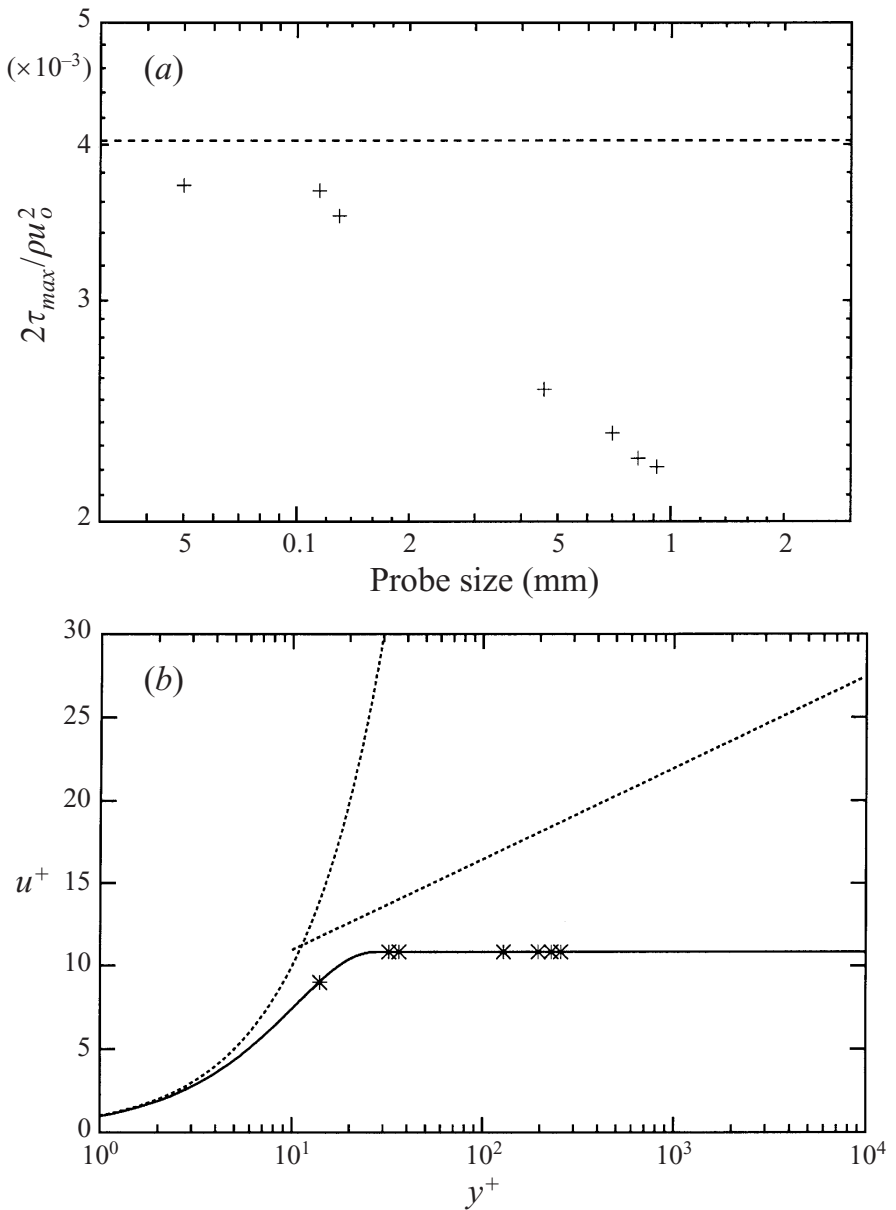

Figure 11. (a) Maximum shear stress under impinging plane jet $\left(H / D=20.6, \operatorname{Re}_{o}=6300\right)$ measured by Tu \& Wood (1996) (+) with various probe sizes compared with predicted laminar value (- - - ), and $(b)$ corresponding probe locations $(*)$ in laminar boundary layer $(-)$ compared with turbulent boundary layer $(\cdots \cdots)$.

to the stagnation point. Various sized Stanton and Preston probes were used to map the wall shear stress distribution under a single impinging plane jet configuration $\left(H / D=20.6, R e_{o}=6300\right)$. The smaller probes yielded consistently higher wall shear stresses along the surface. Figure 11(a) exhibits the measured wall shear stress at the location of maximum shear stress as a function of probe size. The dashed line represents the maximum shear stress value as calculated from equation (2.32). Figure 
11(b) displays the laminar velocity profile at the location of maximum shear stress for the given jet, with the symbols representing the location of the top of the probe within the boundary layer. Again, the correlation between the measurement error and the difference between the laminar and turbulent boundary layer profiles at the probe location is clear. It can be concluded from these analyses that shear stress probes calibrated in turbulent boundary layers yield misleading results close to the stagnation point in an impingement flow. This is significant because the maximum shear stress, a quantity important to the processes of surface cleaning and coating thickness control, is located within this region. The electrochemical method seems to provide the most accurate data close to the stagnation point, but there are no such data available for impinging gas jets. The hot-film and hot-wire measurements of Baines \& Keffer (1976) exhibit large discrepancies close to the stagnation point and are unlikely to be accurate for circular jet impingement due to the three-dimensionality of the flow. The next section presents a unique method to probe wall shear stress under impinging circular gas jets in order to support the theoretical development in $\S 2$ and the previous analyses.

\section{Particle resuspension experiments}

A main motivation of the present work is to predict the conditions under which impinging gas jets produce efficient resuspension of fine particles from surfaces. Usually, the surface needing to be cleaned contains a polydisperse distribution of uncharacterized particles possessing a wide distribution of particle-surface adhesion forces. In this case, knowledge of the wall shear stress distribution under an impinging jet is necessary to predict removal efficiencies. However, if the surface is laden with a uniform distribution of identical spherical particles, then all removal occurs within a very narrow wall shear stress range and the particles behave as quantized shear stress sensors. The removal patterns beneath the jet serve as contours of constant shear stress and can aid in determining the complete shear stress distribution. In these experiments, a particle-laden surface was translated under an impinging air jet so that a larger number of particles could be examined in a single experiment, and no particles were exposed to jet startup transients that might affect resuspension.

\subsection{Apparatus and sample preparation}

The translating jet apparatus, illustrated in figure 12, used in this study is identical to that used in the particle entrainment experiments of Smedley et al. (1999). The system consists of a high-speed rail table with a vacuum-chuck sample holder and a circular jet nozzle mounted to a $y-z$ traverse at the translation point. The rail table motions were controlled via RS-232 communications from the computer to a Compumotor PDX-15 motor controller. The jet pressure is adjusted using the regulator and measured with a Lucas P4100 pressure transducer reported to have an accuracy to $0.03 \%$ of full scale with a range 0 to $6.9 \mathrm{MPa}$ sealed gauge relative to standard atmospheric pressure. The height and horizontal position of the jet are adjusted using linear micrometer stages. A HeNe laser sheet provides glancing angle illumination of the particles on the glass substrate so that they appear bright against a dark background. Dark-field images of the particle-laden surfaces were recorded using a CCD camera equipped with a telephoto lens and connected to a Macintosh computer using a Scion LG3 frame grabber card.

Well-characterized monodisperse particles of polystyrene and ammonium fluorescein were produced with a vibrating orifice aerosol generator (VOAG), specially 


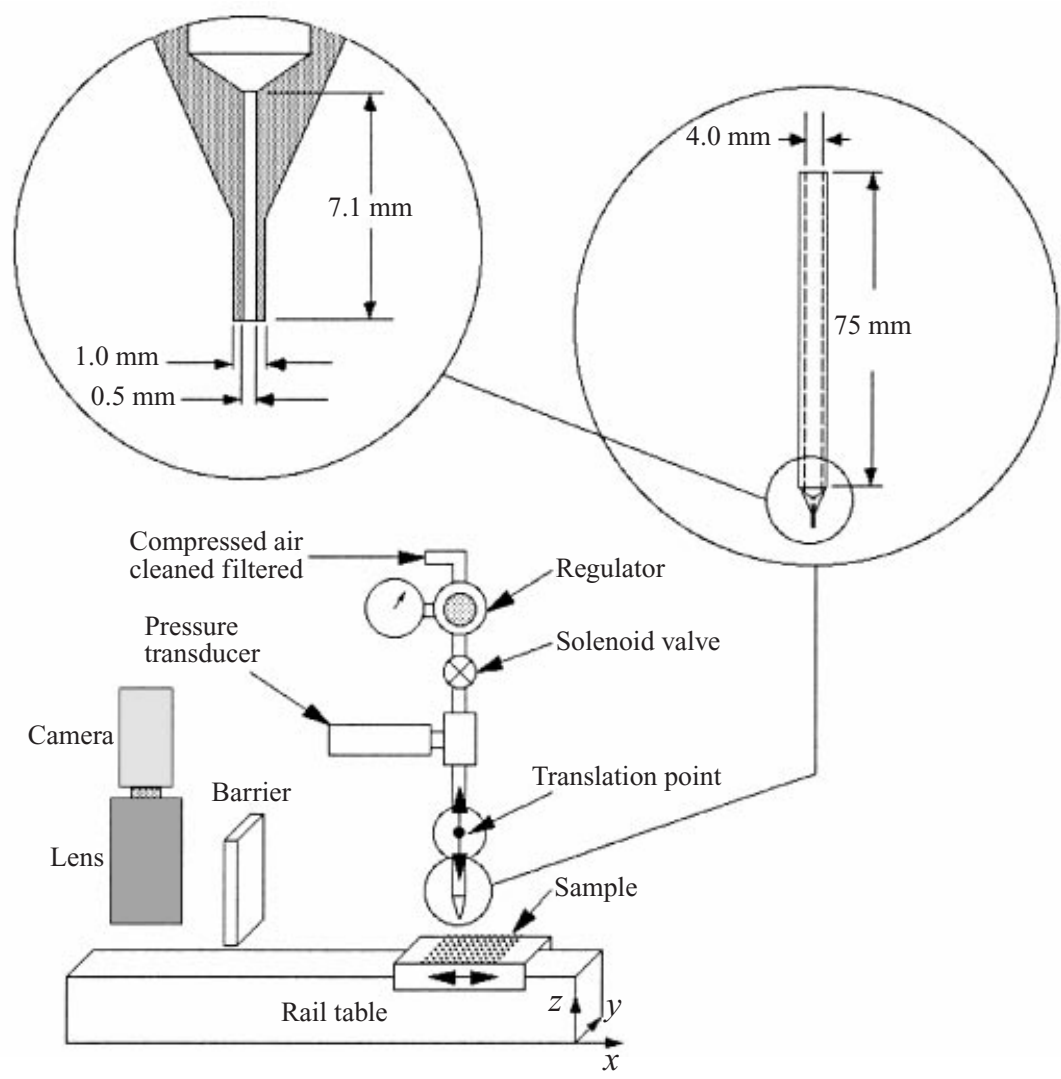

FIGURE 12. Translating gas jet experimental setup.

designed to work with organic solvents (Levendis \& Flagan 1989). The particles were deposited on carefully cleaned microscope slides by gravitational sedimentation and then stored in a dry box for a minimum of 24 hours prior to use in the experiments. The standard deviation for the particles used in this study was less than $0.5 \mu \mathrm{m}$.

\subsection{Procedure}

The procedure and data analysis is described in detail by Smedley et al. (1999), so only the main points will be summarized here. A glass slide with a uniform deposit of monodisperse microspheres $\left(\sim 300\right.$ particles $\left./ \mathrm{mm}^{2}\right)$ was mounted flush on the translation stage and secured with the vacuum chuck. Three dark-field images spanning $58 \mathrm{~mm}$ of the length of the slide were recorded. The gas jet was started and the slide translated at a constant velocity, $V_{\text {trans }}$, under the jet. The slide was then returned to the camera location and three more images were taken to record changes in the average pixel intensity profile across the width of the slide. Particle uniformity ensured that equal light intensity was scattered by each particle and, thus, the average pixel intensity in the images was directly related to the surface density of the deposited particles.

A typical experiment involved passing the slide under the jet 12 times with successively increasing jet pressure ratios, $P_{j e t} / P_{a t m}$. As the jet pressure ratio was increased, an increasing number of particles was entrained from a path directly beneath the jet until $100 \%$ removal was achieved in the path, now resembling a dark stripe. Further 

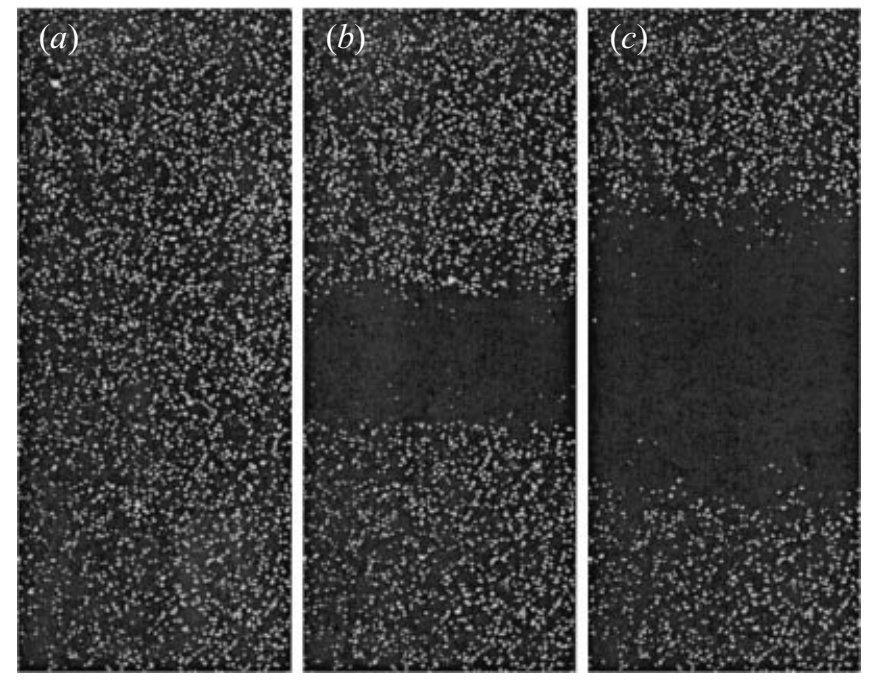

FIGURE 13. Images of Sample 10 recorded $(a)$ before experiment, and after exposure to air jet; (b) $P_{\text {jet }} / P_{\text {atm }}=2.02$, and (c) $P_{\text {jet }} / P_{a t m}=6.71$.

increase in the jet pressure ratio resulted in a widening of the path (see figure 13). The two quantities measured from the recorded images were: (i) the removal efficiency in the removal path passing directly beneath the jet, and (ii) the width, $w$, of the removal path, which was measured between the locations of $50 \%$ removal efficiency.

Since a narrow distribution of particle-surface adhesion forces exists for a given sample, due to slight variation in particle size and non-uniformities in the glass surface, the threshold shear stress for particle resuspension is defined as the shear stress needed to entrain $50 \%$ of the particles exposed to the shear. Thus the threshold shear stress, $\tau_{50 \%}$, is the maximum shear stress under a jet with pressure ratio, $\left(P_{\text {jet }} / P_{a t m}\right)_{50 \%}$, which causes $50 \%$ removal efficiency in the removal path. As the path widens, the removal path half-width, $w / 2$, represents the radial location of the shear stress contour corresponding to $\tau_{50 \%}$ for the subsequent increasing jet pressures. This technique can be used to map the $\tau_{50 \%}$ contour only outside the maximum shear stress location, since all particles within the initial removal path are exposed to the maximum shear stress during translation.

A total of 16 samples were analysed: six $15.7 \mu \mathrm{m}$ ammonium fluorescein particle samples and ten $10.0 \mu \mathrm{m}$ polystyrene particle samples. For the ammonium fluorescein samples, the jet height was held constant $(H / D=10)$ and the translation speed was varied. For the polystyrene samples, the translation speed was held constant $\left(V_{\text {trans }}=1.8 \mathrm{~mm} \mathrm{~s}^{-1}\right)$ and the jet height was varied. Tables 4 and 5 summarize the experimental parameters imposed on the ammonium fluorescein and polystyrene samples, respectively.

\subsection{Results}

\subsubsection{Ammonium fluorescein particles}

Table 4 lists the threshold results for the ammonium fluorescein particle samples. The threshold shear stresses, $\tau_{50 \%}$, were calculated from the threshold jet pressure ratio, $\left(P_{\text {jet }} / P_{a t m}\right)_{50 \%}$, and jet dynamic pressure, $\left(\rho u_{o}^{2}\right)_{50 \%}$, using equation (2.33). The threshold shear stress increase with translation speed suggests an effect of force duration on the threshold particle resuspension force. This dynamic property of 


\begin{tabular}{cccccc}
\multicolumn{1}{c}{} & $V_{\text {trans }}$ & & $\left(\rho u_{o}^{2}\right)_{50 \%}$ & $\tau_{50 \%}$ \\
Sample & $\left(\mathrm{mm} \mathrm{s}^{-1}\right)$ & $\left(P_{\text {jet }} / P_{\text {atm }}\right)_{50 \%}$ & $(\mathrm{kPa})$ & $\operatorname{Re}_{50 \%}$ & $(\mathrm{~Pa})$ \\
1 & 1.8 & 1.15 & 2.85 & 5031 & 179 \\
2 & 1.8 & 1.16 & 3.05 & 5211 & 189 \\
3 & 9.0 & 1.21 & 3.99 & 5959 & 231 \\
4 & 9.0 & 1.19 & 3.69 & 5728 & 217 \\
5 & 270.0 & 1.24 & 4.46 & 6300 & 251 \\
6 & 270.0 & 1.25 & 4.66 & 6440 & 259
\end{tabular}

TABLE 4. Experimental parameters and threshold results for $15.7 \mu \mathrm{m}$ ammonium fluorescein particle samples $(H / D=10)$.

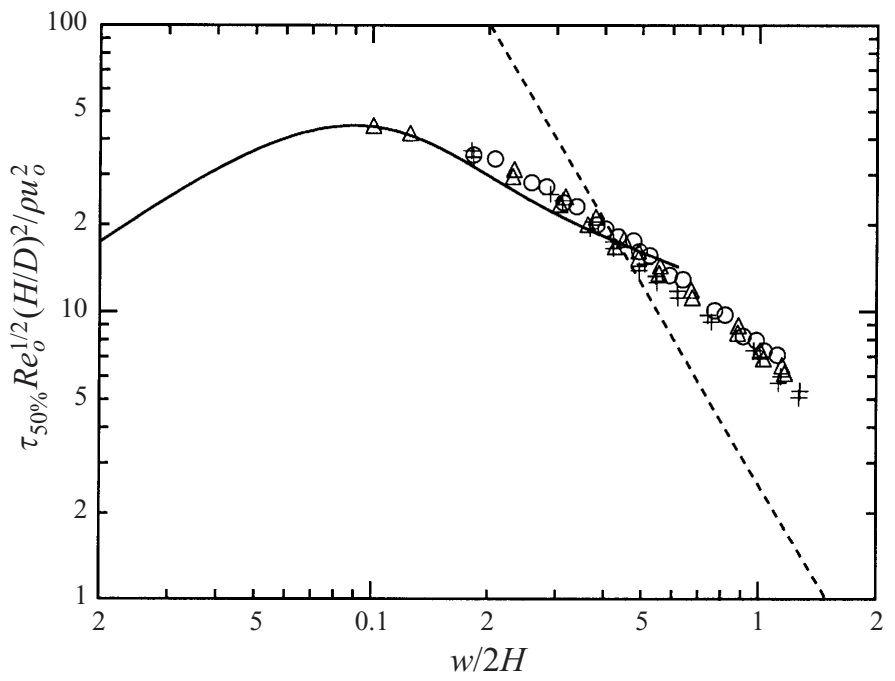

FIGURE 14. Observed $\tau_{50 \%}$ shear stress locations for $V_{\text {trans }}=1.8 \mathrm{~mm} \mathrm{~s}^{-1}(+), V_{\text {trans }}=9.0 \mathrm{~mm} \mathrm{~s}^{-1}(\mathrm{O})$, and $V_{\text {trans }}=270.0 \mathrm{~mm} \mathrm{~s}^{-1}(\triangle)$ compared with impingement-region model $(-)$, and wall-jet-region expression (equation (2.37)) (- - -).

particle resuspension allowed different shear stress contours to be examined with identical test samples by varying the translation speed.

Figure 14 presents the shear stress distribution, scaled with the appropriate jet exit conditions, for all of the ammonium fluorescein test samples. The predicted impingement-region shear stress distribution is represented by a solid line; and the wall-jet-region distribution by a dashed line. Despite the variation in threshold shear stress for the various translation speeds used, all of the data collapse to the curve predicted by the impingement-region model close to the maximum shear stress location. Farther from the stagnation point, the data lie above the wall-jet-region shear stress curve. This suggests that particle resuspension scales with some percentage of the amplitude of the wall shear stress fluctuations, which increases significantly at the turbulent transition location.

\subsubsection{Polystyrene particles}

The smaller polystyrene particles required higher jet pressure ratios to achieve removal efficiencies comparable to those observed for the ammonium fluorescein 


$\begin{array}{cccccc}\text { Sample } & H / D & \left(P_{j e t} / P_{a t m}\right)_{50 \%} & \begin{array}{c}\left(\rho u_{o}^{2}\right)_{50 \%} \\ (\mathrm{kPa})\end{array} & R e_{50 \%} & M_{50 \%} \\ 7 & 3 & 1.17 & 1.84 & 4182 & 0.36 \\ 8 & 4 & 1.08 & 1.46 & 3708 & 0.32 \\ 9 & 6 & 1.09 & 1.48 & 3726 & 0.32 \\ 10 & 10 & 1.20 & 3.67 & 6103 & 0.52 \\ 11 & 10 & 1.24 & 4.39 & 6764 & 0.56 \\ 12 & 10 & 1.21 & 3.97 & 6389 & 0.53 \\ 13 & 12 & 1.36 & 6.46 & 8482 & 0.68 \\ 14 & 15 & 1.45 & 7.96 & 9640 & 0.75 \\ 15 & 17 & 1.54 & 9.28 & 10623 & 0.81 \\ 16 & 20 & 1.62 & 10.49 & 11496 & 0.86\end{array}$

TABLE 5. Experimental parameters and threshold results for $10.0 \mu \mathrm{m}$ polystyrene particle samples $\left(V_{\text {trans }}=1.8 \mathrm{~mm} \mathrm{~s}^{-1}\right)$.

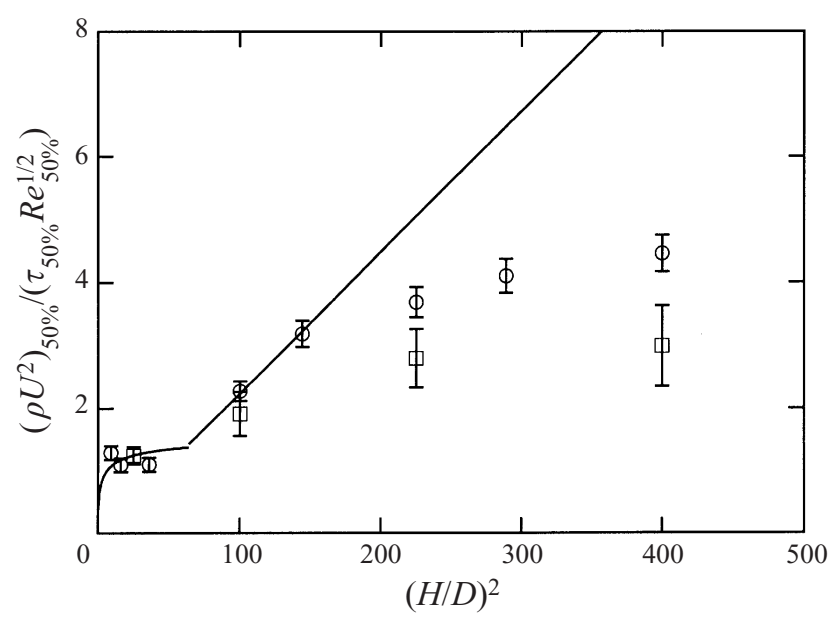

FIGURE 15. Comparison between measured threshold jet parameters (present experiment $(O)$ and experiment of Smedley et al. $1999(\square)$ ) and incompressible flow theory (-).

particles. For the higher jet heights, the threshold jet pressure ratios produced compressible jets, which were not considered in $\S 2$. Although large jet pressure ratios were used in the ammonium fluorescein experiments, particle removal had already extended far into the wall jet region of the flow and, thus, the effect of compressibility could not be studied. In the polystyrene experiments, threshold jet pressure ratios, $\left(P_{\text {jet }} / P_{a t m}\right)_{50 \%}$, produced compressible jets, facilitating investigation of the effect of compressibility on impingement-region wall shear stress. Table 5 lists the threshold results measured for each case, including the threshold Mach number at the jet exit, $M_{50 \%}$.

Since translation velocity was held constant, the threshold shear stress, $\tau_{50 \%}$, was assumed to be the same for all of the polystyrene samples. This notion was tested with three repeatability experiments run at the same jet height $(H / D=10)$ on Samples 10,11 , and 12 . Only a $3 \%$ variablity in $\left(P_{\text {jet }} / P_{\text {atm }}\right)_{50 \%}$ was measured, supporting the assumption of threshold shear stress uniformity among all samples.

The variation in measured threshold jet conditions with jet height is presented in figure 15 along with the predictions from incompressible flow theory. The data show 


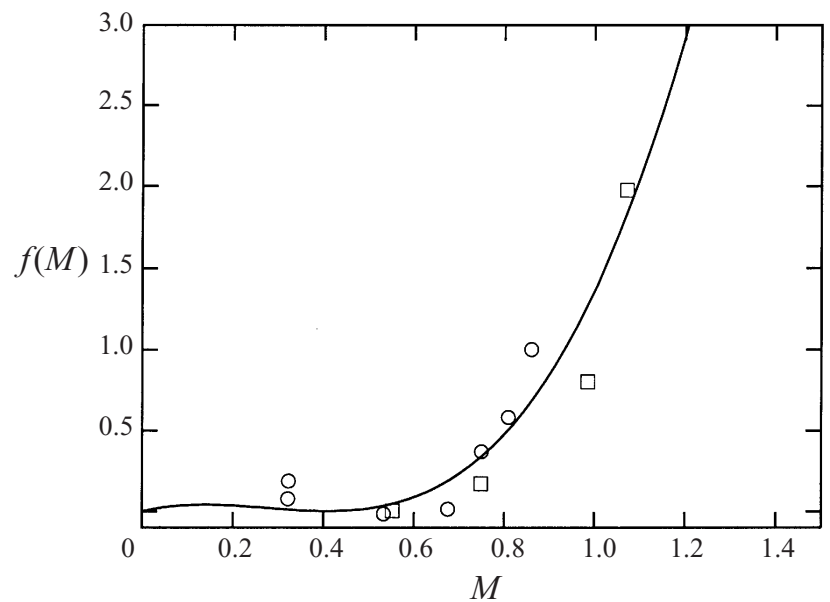

FIGURE 16. Measured Mach number correction function (present experiment $(O)$ and Smedley et al. (1999) ( $\square$ )) with overlaid polynomial fit (equation (4.2)) ( $\square$ ).

a definite trend away from the incompressible model for increasing jet heights. The measured threshold jet conditions of Smedley et al. (1999) for $8.3 \mu \mathrm{m}$ polystyrene particles, also plotted in figure 15, exhibit a similar deviation from the incompressible model, but for even lower jet heights. This suggests that the deviation is indeed due to jet compressibility.

To account for the deviation, we introduce a compressibility correction factor to equation (2.33):

$$
\tau_{m}=44.6 \rho u_{o}^{2} R e_{o}^{-0.5}\left(\frac{H}{D}\right)^{-2}[1+f(M)],
$$

where $f(M)$ is some function of the Mach number. Figure 16 displays the values of $f(M)$ calculated from the present data set and from the measurements of Smedley et al. (1999). The data were fitted to a fourth-order polynomial to obtain an empirical expression for the compressibility correction factor in an impinging air jet given by

$$
f(M)=0.66 M-3.2 M^{2}+3.9 M^{3} .
$$

\section{Concluding remarks}

The assumption of a fully developed turbulent boundary layer close to the stagnation point of an impinging jet has led to inaccuracies in the description of the wall shear stress distribution. Reported measurements using pressure probes calibrated in fully developed turbulent boundary layers have included errors in the magnitude and location of the maximum wall shear stress, two quantities which are critical for predicting shear-induced resuspension for surface cleaning or particulate sampling applications. Similarly, numerical solutions (Looney \& Walsh 1984; Bouainouche et al. 1997) of the impinging jet flow field have also assumed a fully developed turbulent boundary layer throughout the impingement region by employing turbulent wall functions as the surface boundary condition, resulting in underpredictions of the mean shear stress in the impingement region. A laminar boundary layer analysis was employed to describe discrepancies between previously reported wall shear stress measurements for jet Reynolds numbers as high as $R e_{o}=10^{5}$. The laminarization of 
the boundary layer close to the stagnation point is analogous to the relaminarization of a turbulent boundary layer in a strong favourable pressure gradient (Patel 1965). Observing the removal of monosized spheres to map out wall shear stress contours also yielded results which corroborated the predictions of laminar boundary layer theory. This technique was applied to impinging compressible jets, and the wall shear stress rise due to compressibility effects was measured for various Mach numbers. An empirical expression relating the wall shear stress maximum and the jet Mach number was obtained for impinging air jets.

Support for this work was provided by the FAA under Grant 93-G-060.

\section{REFERENCES}

Abramovich, G. N. 1963 The Theory of Turbulent Jets. The MIT Press.

Alekseenko, S. V. \& Markovich, D. M. 1994 Electrodiffusion diagnostics of wall shear stresses in impinging jets. J. Appl. Electrochem. 24, 626-631.

BAINES, W. D. \& KefFER, J. F. 1976 Shear stress and heat transfer at a stagnation point. Intl J. Heat Mass Transfer 19, 21-26.

Beltaos, S. \& Rajaratnam, N. 1973 Plane turbulent impinging jets. J. Hydr. Res. 11, 29-59.

Beltaos, S. \& Rajaratnam, N. 1974 Circular turbulent impinging jets. J. Hyd. Div., ASCE 100, $1313-1328$.

Bouainouche, M., BourabaA, N. \& Desmet, B. 1997 Numerical study of the wall shear stress produced by the impingement of a plane turbulent jet on a plate. Intl J. Numer. Meth. Heat Fluid Flow 7, 548-564.

Bradbury, L. J. S. 1972 The impact of an axisymmetric jet onto a normal ground. Aero. Q. 23, $141-147$.

Bradshaw, P. \& Love, E. M. 1961 The normal impingement of a circular air jet over a flat surface. $A R C R \& M 3205$.

Champion, M. \& Libby, P. A. 1994 Reynolds stress description of opposed and impinging turbulent jets. II. Axisymmetric jets impinging on nearby walls. Phys. Fluids 6, 1805-1819.

Deshpande, M. D. \& Vaishnav, R. N. 1982 Submerged laminar jet impingement on a plane. $J$. Fluid Mech. 114, 213-236.

Giralt, F., Chia, C. \& Trass, O. 1977 Characterization of the impingement region in an axisymmetric turbulent jet. Ind. Engng Chem. Fundam. 16, 21-28.

Glauert, M. B. 1956 The wall jet. J. Fluid Mech. 1, 625-643.

Kataoka, K., Kamiyama, Y., Hashimoto, S. \& Komai, K. 1982 Mass transfer between a plane surface and an impinging jet. J. Fluid Mech. 119, 91-105.

KataOKa, K. \& Mizushina, T. 1974 Local enhancement of the rate of heat transfer in an impinging round jet by free-stream turbulence. In Proc. 5th Intl Heat Transfer Conf., FC8.3.

Kumada, M. \& Mabuchi, I. 1970 Studies on the heat transfer of impinging jets. Bull. JSME, 13, $77-85$.

Lauder, B. E. \& Rodi, W. 1983 The turbulent wall jet - measurements and modeling. Ann. Rev. Fluid Mech. 15, 429-459.

Levendis, Y. A. \& Flagan, R. C. 1989 Synthesis, formation, and characterization of micron-sized glassy-carbon spheres of controlled pore structure. Carbon 27, 265-283.

LOONEY, M. K. \& WALSH, J. J. 1984 Mean-flow and turbulent characteristics of free and impinging jet flows. J. Fluid Mech. 147, 397-429.

PATEL, V. C. 1965 Calibration of the Preston tube and limitations on its use in pressure gradients. J. Fluid Mech. 23, 185-208.

Phares, D. J., Holt, J. K., Smedley, G. T. \& Flagan, R. C. $2000 a$ Method for characterization of adhesion properties of trace explosives in fingerprints and fingerprint simulations. J. Forensic Sci. 45, 762-772.

Phares, D. J., Smedley, G. T. \& Flagan, R. C. $2000 b$ The inviscid impingement of a jet with arbitrary velocity profile. Phys. Fluids, 12, 2046-2055. 
Poreh, M., Tsuei, Y. G. \& Cermak, J. E. 1967 Investigation of a turbulent radial wall jet. Trans. ASME: J. Appl. Mech. 34, 457-463.

Rubel, A. 1980 Computations of jet impingement on a flat surface. AIAA J. 18, 168-175.

Rubel, A. 1983 Inviscid axisymmetric jet impingement with recirculating stagnation regions. $A I A A$ J. 21, 351-357.

Schauer, J. J. \& Eustis, R. H. 1963 The flow development and heat transfer characteristics of plane turbulent impinging jets. Tech. Rep., Dept. Mech. Eng., Stanford, CA.

Schlichting, H. 1960 Boundary Layer Theory. McGraw-Hill.

ScholtZ, M. T. \& Trass, O. 1970 Mass transfer in a nonuniform impinging jet. AIChE J. 16, 82-90.

Smedley, G. T., Phares, D. J. \& Flagan, R.C. 1999 Entrainment of fine particles from surfaces by gas jets impinging at normal incidence. Exps. Fluids 26, 324-334.

STRAND, T. 1964 On the theory of normal ground impingement of axisymmetric jets in inviscid incompressible flow. AIAA Paper 64-424.

Tu, C. V. \& WooD, D. H. 1996 Wall pressure and shear stress measurements beneath an impinging jet. Expl Therm. Fluid Sci. 13, 364-373.

Wall, T. F., Subramanian, V. \& Howley, P. 1982 An experimental study of the geometry, mixing and entrainment of particle-laden jets up to ten diameters from the nozzle. Trans. Inst. Chem. Engrs 60, 231-239. 